Pacific Journal of Mathematics

RANDOM CROSSINGS OF CUMULATIVE DISTRIBUTION 


\title{
RANDOM CROSSINGS OF CUMULATIVE DISTRIBUTION FUNCTIONS
}

\author{
MeYer DWASS
}

1. Introduction. Let $X_{1}, \cdots, X_{n}$ be $n$ independent and identically distributed random variables, each with continuous c.d.f. (cumulative distribution function), $F(x)$. Let $F_{n}(x)$ be the empirical c.d.f. of the $n$ random variables and let $N_{1}(n)$ be the number of times $F_{n}$ equals $F$. There is no loss of generality in supposing that the $X_{i}$ 's are distributed uniformly over the interval $(0,1)$, and to be specific, $N_{1}(n)$ is defined by

$$
N_{1}(n)=\text { number of indices, } i \text {, for which } F_{n}(i / n)=i / n, \quad i=1, \cdots, n \text {. }
$$

Similary, let $X_{1}, \cdots, X_{n}, \cdots, Y_{1}, \cdots, Y_{n}$ be $2 n$ independent random variables, each with the same continuous c.d.f., $F(x)$, and let $F_{n}, G_{n}$ denote the empirical c.d.f.'s of the $X_{i}$ 's and $Y_{i}$ 's respectively. Let $N_{2}(n)$ be the number of times $F_{n}$ equals $G_{n}$. That is.

$$
\begin{gathered}
N_{2}(n)=\text { number of indices } i \text { for which } F_{n}\left(X_{i}\right)=G_{n}\left(X_{i}\right), \\
\text { plus }
\end{gathered}
$$

number of indices $i$ for which $F_{n}\left(Y_{i}\right)=G_{n}\left(Y_{i}\right), \quad i=1, \cdots, n$.

The purpose of this paper is to show that

$$
\lim _{n \rightarrow \infty} P\left(\frac{N_{1}(n)}{\sqrt{2 n}}<t\right)=\lim _{n \rightarrow \infty} P\left(\frac{N_{2}(n)}{\sqrt{4 n}}<t\right)=1-e^{-t^{2}} .
$$

The methods for obtaining these results are practically the same for $N_{1}$ and $N_{2}$, so the first case is treated with somewhat greater detail. In both cases, the random variables are related to other random variables on appropriate stochastic processes with independent increments, to obtain generating functions for the moments of $N_{i}$. The Karamata Tauberian theorem is then applied to describe the asymptotic behavior of these moments.

2. Some preliminaries on the Poisson process. Let $Y(t)$ be the Poisson process with stationary independent increments, $t \geqq 0, Y(0)=0$, $E[Y(1)]=1$. Consider $\gamma t$, the straight line coming out of the origin with slope $\gamma>1$. The random function $Y(t)$ can equal $\gamma t$ at times $1 / \gamma$, $2 / \gamma$, etc. The event that $Y(t)=\gamma t$ is a recurrent event in the sense of Feller [4]. Because $\gamma$ is greater than 1, this recurrent event is an uncertain one. It was shown by Baxter and Donsker [1] that

Received February 23, 1960. Research done under contract with the U.S. Office of Naval Research. Contract Nonr-1228(10), project NR 047-021. 


$$
P[Y(t)<\gamma t \text {, all positive } t]=1-1 / \gamma .
$$

A completely elementary proof of this fact was given by Dwass [3]. In other words, the probability that the uncertain recurrent event under discussion never takes place is $1-1 / \gamma$. To introduce some specific notation, let $N=$ number of times that $Y(t)$ equals $\gamma t$. That is,

$$
N=\text { number of indices } i \text { for which } Y(i / \gamma)=\gamma(i / \gamma)=i, \quad i=1,2, \cdots
$$

The random variable, $N$ is geometrically distributed, specificially,

$$
P(N=k)=(1 / \gamma)^{k}(1-1 / \gamma),
$$

and for the $r$ th factorial moment we have,

$$
E N^{(r)}=E N(N-1)(N-2) \cdots(N-r+1)=r ! /(r-1)^{r} .
$$

3. A generating function for $E\left[N_{1}^{(r)}(n)\right]$. The link between the random variables $N$ and $N_{1}(n)$ lies in the following lemma.

LEMma 3.1. The conditional distribution of $N$ given that $Y(t)=\gamma t$ for the last time at time $t=n / \gamma$ is exactly the same as the distribution of $N_{1}(n)$.

Proof of Lemma 3.1. This follows directly from the well-known fact that the places where the jumps of $Y(t)$ occur in the interval $(0, a)$ are distributed as $n$ randomly chosen points in $(0, a)$ under the condition that $Y(a)=n$.

Making use of this lemma, we can compute the $r$ th factorical moment of $N$ in the following iterative way. Let $A_{k}$ denote the event that the last crossing of $\gamma t$ by $Y(t)$ takes place at time $k / \gamma$. Then

$$
E\left(N^{(r)}\right)=\sum_{k=0}^{\infty} E\left(N^{(r)} \mid A_{k}\right) P\left(A_{k}\right) \text {. }
$$

Since

$$
P\left(A_{k}\right)=(k / \gamma)^{k} e^{-k / \gamma}(1-1 / \gamma) / k !
$$

and

$$
E\left(N^{(r)} \mid A_{k}\right)=E\left[N_{1}^{(r)}(k)\right],
$$

we have, making use of (2.1), the following theorem.

\section{THEOREM.}

$$
\sum_{k=0}^{\infty} \frac{e^{-k} k^{k}}{k !} E N_{1}^{(r)}(k)\left(\frac{e^{1-1 / \gamma}}{\gamma}\right)^{k}=\frac{r ! \gamma}{(\gamma-1)^{r+1}} .
$$

REMARKS.

(a) In (3.1), $e^{-k} k^{k} / k$ ! should be understood to be 1 when $k=0$. 
(b) $u=e^{1-1 / \gamma}$ is a strictly decreasing function of $1 / \gamma$, for $\gamma \geqq 1$, and maps $(1, \infty)$ onto $(0,1)$. Let $1 / \gamma=P(u)$ denote the inverse function. Then (2.2) can be rewritten,

$$
\begin{aligned}
& \sum_{k=0}^{\infty} \frac{e^{-k} k^{k}}{k !} E N_{1}^{(r)}(k) u^{k}=\frac{r !}{P(u)\left[P^{-1}(u)-1\right]^{r+1}}=h(u), \\
& 0 \leqq u<1 .
\end{aligned}
$$

Since

$$
\lim _{x \rightarrow 1} \frac{1-x e^{1-x}}{(x-1)^{2}}=1 / 2,
$$

or equivalently,

$$
\lim _{u \rightarrow 1} \frac{1-u}{\left(P^{-1}(u)-1\right)^{2}}=1 / 2,
$$

it follows that

$$
\lim _{u \rightarrow 1}(1-u)^{(r+1) / 2} h(u)=\frac{r !}{2^{(r+1) / 2}} .
$$

If the coefficients of $u^{k}$ in $h(u)$ form an increasing sequence, then Karamata's Tauberian theorem is applicable and we could conclude that the sum of the first $k$ coefficients of powers of $u$ in $(1-u) h(u)$ is asymptotically equal to

$$
k^{(r-1) / 2} \frac{r !}{2^{(r+1) / 2} \Gamma\left(\frac{r+1}{2}\right)},
$$

or equivalently,

$$
\begin{gathered}
\lim _{k \rightarrow \infty} \frac{\frac{e^{-k} k^{k}}{k !} E N_{1}^{(r)}(k)}{k^{(r-1) / 2}}=\frac{r !}{2^{(r+1) / 2} \Gamma\left(\frac{r+1}{2}\right)} \\
\lim _{k \rightarrow \infty} \frac{E N_{1}^{(r)}(k)}{k^{r / 2}}=\frac{r ! \sqrt{\pi}}{2^{r / 2} \Gamma\left(\frac{r+1}{2}\right)}=2^{r / 2} \Gamma\left(\frac{r}{2}+1\right)
\end{gathered}
$$

by the "duplication formula" for the gamma function (p. 240 [6]).

Since the asymptotic behavior of the $r$ th factorial moment is the same as that of the rth ordinary moment, we would have finally,

$$
\lim _{k \rightarrow \infty} E\left(\frac{N_{1}(k)}{\sqrt{2 k}}\right)^{r}=\Gamma\left(\frac{r}{2}+1\right)=\int_{0}^{\infty} x^{r} f(x) d x
$$

where $f(x)$ is the probability density function 


$$
f(x)=\left\{\begin{array}{ll}
2 x e^{-x^{2}}, & x \geqq 0 \\
0, & x<0
\end{array},\right.
$$

However, it is not at all clear that the usual conditions for Karamata's theorem to hold are applicable, and a slightly more delicate argument is required.

4. The limiting distribution of $N_{1}(n)$. Following the discussion in the last section, the main effort which remains is to justify the applicability of Karamata's theorem.

LemmA 4.1. Let $a_{i}(u),(i=1, \cdots, r)$ be power series having positive, non-decreasing coefficients. Then $a(u)=\Pi_{i} a_{i}(u)$ has the same property.

Proof of Lemma 4.1. $a_{i}(u)$ has positive, non-decreasing coefficients means that the coefficients of $(1-u) a_{i}(u)$ are non-negative.

$$
(1-u) \prod_{i} a_{i}(u)=\prod_{i}\left[(1-u) a_{i}(u)\right](1-u)^{-(r-1)}
$$

is a product of power series all with non-negative coefficients, which completes the proof.

LEMMA 4.2 .

$$
\begin{gathered}
\sum_{k=0}^{\infty} \frac{k^{k} e^{-k}}{k !} u^{k}=f(u)=1 /(\gamma-1) \\
c(1-u)^{-1 / 2}-f(u)=g(u)
\end{gathered}
$$

is a power series with positive, non-decreasing coefficients if $c$ is suffciently large.

Proof of Lemma 4.2. Part (a) follows from (3.1) for $r=0$. The coefficients of $(1-u)^{-1 / 2}$ are of the order of $1 / \sqrt{k}$ and strictly positive. The coefficients of $-f(u)$ are strictly increasing and also of the order of $1 / \sqrt{k}$. Hence choosing $c$ sufficiently large will guarantee the result.

Finally, we want to state the following form of Karamata's theorem.

LEMMA 4.3. Let $a(u)=\sum_{k=0}^{\infty} a_{k} u^{k}$ where $\left\{a_{k}\right\}$ is a non-decreasing sequence, and suppose $(1-u)^{\gamma} a(u) \rightarrow A$ as $u \rightarrow 1$, for $\gamma \geqq 0$. Then

$$
\frac{a^{k}}{k^{\gamma-1}} \rightarrow \frac{A}{\Gamma(\gamma)}
$$

as $k \rightarrow \infty$.

Proof of Lemma 4.3. For $\gamma>1$ the result follows from the con- 
ventional form of Karamata's theorem (for example see Theorem 4.3, p. 192, [5]) by considering that

$$
(1-u)^{\gamma-1}(1-u) a(u) \rightarrow A
$$

and that the partial sums of coefficients in $(1-u) a(u)$ are $a_{k}$.

For $0 \leqq \gamma<1$ we have that

$$
a_{1}+\cdots+a_{k} \sim \frac{A}{\Gamma(\gamma+1)} k^{\gamma}
$$

and we can apply Hilfassatz 3, p. 517, Doetsch, [2], to conclude that

$$
a_{k} \sim \frac{A \gamma}{\Gamma(\gamma+1)} k^{\gamma}=\frac{A}{\Gamma(\gamma)} k^{\gamma} .
$$

We can now prove the following.

THEOREM.

$$
\lim _{n \rightarrow \infty} P\left(\frac{N_{1}(n)}{\sqrt{2 n}}<t\right)=2 \int_{0}^{t} x e^{-x^{2}} d x=1-e^{-t^{2}}
$$

Proof. The limiting distribution is determined by its moments, hence it is sufficient to show that

$$
\lim _{n \rightarrow \infty} E\left(\frac{N_{1}(n)}{\sqrt{2 n}}\right)^{r}=2 \int_{0}^{\infty} x^{r+1} e^{-x^{2}} d x=\Gamma(r / 2+1), \quad r=1,2, \cdots .
$$

Referring to (3.2) and to Lemma 4.2, we can write

$$
\begin{aligned}
h(u) & =r ![1+f(u)][f(u)]^{r} \\
& =\left[c(1-u)^{-1 / 2}-g(u)+1\right]\left[c(1-u)^{-1 / 2}-g(u)\right]^{r} .
\end{aligned}
$$

Since $g(u)$ has positive and increasing coefficients then by Lemma 4.1 so does $(1-u)^{-m / 2}[g(u)]^{n}$ for $m, n$ positive integers, because

$$
(1-u)\left[(1-u)^{-m / 2}(g(u))^{n}\right]=(1-u)^{-m / 2}(1-u)[g(u)]^{n}
$$

has positive coefficients. Hence by Karamata's theorem, since

$$
(1-u)^{(m+n) / 2}(1-u)^{-m / 2}[g(u)]^{n} \rightarrow\left(c-\frac{1}{\sqrt{2}}\right)^{n},
$$

the coefficients of $(1-u)^{-m / 2}[g(u)]^{n}$ are asymptotically equivalent to

$$
\frac{(c-1 / \sqrt{2})^{n}}{\Gamma\left(\frac{m+n}{2}\right)} k^{(m+n) / 2} .
$$

On expanding the right side of (4.1), an elementary computation yields 
the result that the coefficients of $h(u)$ are asymptotically equivalent to

$$
\frac{r !}{2^{(r+1) / 2} \Gamma\left(\frac{r+1}{2}\right)} k^{(r-1) / 2} .
$$

According to the discussion in $\S 4$, we conclude then that

$$
\lim _{k \rightarrow \infty} E\left(\frac{N_{1}(k)}{\sqrt{2 k}}\right)^{r}=\Gamma\left(\frac{r}{2}+1\right),
$$

which completes the proof of the theorem.

5. The limiting distribution of $N_{2}(n)$. In this section we prove the following.

THEOREM.

$$
\lim _{n \rightarrow \infty} P\left(\frac{N_{2}(n)}{\sqrt{4 n}}<t\right)=1-e^{-t^{2}}
$$

The main points of the proof are essentially the same as in the preceding theorem, so we offer an outline of the method only.

Let $X_{1}, X_{2}, \cdots$ be a sequence of independent, identically distributed random variables such that

$$
X_{i}=\left\{\begin{array}{l}
1 \text { with probability } p \\
0 \text { with probability } 1-p .
\end{array}\right.
$$

and let $S_{n}$ denote the sum of the first $n$ random variables.

The event that for a positive integer $n, S_{2 n}=n$, is a well-known recurrent event, representing return to the origin, in a discrete random walk on the line. Suppose $p<1 / 2$. Then the probability that the recurrent event never takes place is $1-2 p$. (See Feller, p. 288, [4].) Using $N$ exactly as above, let $N=$ number of indices $i$ for which $S_{2 i}=i$, $i=1,2, \cdots$. As before, $N$ is a geometric random variable, such that

$$
P(N=k)=(1-2 p)(2 p)^{k},
$$

and hence

$$
E N^{(r)}=\frac{r !}{\left(\frac{1}{2 p}-t\right)^{r}} .
$$

Analogous to Lemma 3.1 is the following combinatorial lemma.

LEMma 5.1. The conditional distribution $N$ given that $S_{2 i}=i$ for the last time when $i=n$ is exactly the same as the distribution of 
$N_{2}(n)$. We omit the proof which is elementary. Then

Let $A_{k}$ denote the event that $S_{2 i}=i$ for the last time when $i=k$.

$$
\begin{aligned}
E N^{(r)} & =\sum_{k=0}^{\infty} E\left(N^{(r)} \mid A_{k}\right) P\left(A_{k}\right) \\
& =\sum_{k=0}^{\infty} E N_{2}^{(r)}(k)\left(\begin{array}{c}
2 k \\
k
\end{array}\right) p^{k}(1-p)^{k}(1-2 p) .
\end{aligned}
$$

Hence

$$
f(u)=\sum_{k=0}^{\infty} E N_{2}^{(r)}(k)\left(\begin{array}{c}
2 k \\
k
\end{array}\right) 4^{k} u^{k}=\frac{r !}{2 h(u)\left(\frac{1}{2 h(u)}-1\right)^{r+1}}
$$

where $4 p(1-p)=u, 0 \leqq p \leqq 1 / 2$, is an increasing function of $p$ which maps $(0,1 / 2)$ onto $(0,1)$, and where $p=h(u)$ is the inverse function.

We next notice that

$$
\lim _{u \rightarrow 1}(1-u)^{(r+1) / 2} f(u)=r !,
$$

This follows from the fact that

$$
\lim _{p \rightarrow 1} \frac{1-4 p(1-p)}{\left(\frac{1}{2 p}-1\right)^{2}}=1
$$

The application of the Karamata theorem can now be justified exactly as before. In fact if $g(u)$ is defined in terms of $f(u)$ as in $\S 4$, then the details go through exactly word for word. Hence we conclude that

$$
\lim _{k \rightarrow \infty} E \frac{N_{2}^{(r)}(k)\left(\begin{array}{c}
2 k \\
k
\end{array}\right) 4^{-k}}{k^{(r-1) / 2}}=\frac{r !}{\Gamma\left(\frac{r+1}{2}\right)}
$$

hence

$$
\lim _{k \rightarrow \infty} E \frac{N_{2}^{(r)}(k)}{(4 k)^{r / 2}}=\frac{r ! \sqrt{\pi}}{2^{r} \Gamma\left(\frac{r+1}{2}\right)}=\Gamma\left(\frac{r}{2}+1\right)
$$

which completes the proof.

6. Final remarks. The asymptotic distribution of $N_{1}(n)$ has been studied by N. V. Smirnov in "Sur les écarts de la courbe de distribution empirique", Mat. Sbornik, 6 (48), pp. 3-26 (1939), (Russian, French summary). His methods are not based on the Karamata Tauberian 
theorem and seem considerably more complicated than those of this paper, though he actually dealt with a more general situation. Also, the referee has kindly pointed out that the random variable $N_{2}(n)$ is related to a random variable studied by W. Feller in "The number of zeros and of changes of sign in a symmetric random walk", L'Enseignement Mathématique, III, 3, (1957), 229-235.

\section{REFERENCES}

1. G. Baxter and M.D. Donsker, On the distribution of the supremum functional for processes with stationary independent increments, Trans. Amer. Math. Soc., 85 (1957), 7387.

2. G. Doetsch, Handbuch der Laplace-Transformation, Band I, Basel: Birkhäuser Verlag, 1950 .

3. Meyer Dwass, The distribution of a generalized $D_{n}^{+}$statistic, Ann. Math. Stat. 30, December, (1958), 1024-1028.

4. William Feller, An introduction to probability theory and its applications, 2nd edition, New York: John Wiley and Sons, 1957.

5. D. V. Widder, The Laplace Transform, Princeton: Princeton University Press, 1946.

6. E. T. Whittaker, and G. N. Watson, A Course of Modern Analysis, 4th ed. Cambridge: Cambridge Press, 1950.

NORTHWESTERN UNIVERSITY 


\section{PACIFIC JOURNAL OF MATHEMATICS}

\section{EDITORS}

Ralph S. Phillips

Stanford University

Stanford, California

F. H. BrownelL

University of Washington

Seattle 5 , Washington
A. L. Whiteman

University of Southern California Los Angeles 7. California

L. J. PAIGE

University of California

Los Angeles 24, California

ASSOCIATE EDITORS
E. F. BECKENBACH
D. DERRY
H. L. ROYDEN
E. G. STRAUS
T. M. CHERRY
M. OHTSUKA
E. SPANIER
F. WOLF

\section{SUPPORTING INSTITUTIONS}

UNIVERSITY OF BRITISH COLUMBIA

CALIFORNIA INSTITUTE OF TECHNOLOGY

UNIVERSITY OF CALIFORNIA

MONTANA STATE UNIVERSITY

UNIVERSITY OF NEVADA

NEW MEXICO STATE UNIVERSITY

OREGON STATE COLLEGE

UNIVERSITY OF OREGON

OSAKA UNIVERSITY

UNIVERSITY OF SOUTHERN CALIFORNIA
STANFORD UNIVERSITY

UNIVERSITY OF TOKYO

UNIVERSITY OF UTAH

WASHINGTON STATE COLLEGE,

UNIVERSITY OF WASHINGTON

AMERICAN MATHEMATICAL SOCIETY

CALIFORNIA RESEARCH CORPORATION

HUGHES AIRCRAFT COMPANY

SPACE TECHNOLOGY LABORATORIES

NAVAL ORDNANCE TEST STATION

Printed in Japan by International Academic Printing Co., Ltd., Tokyo, Japan

Reprinted 1966 in the United States of America 


\section{Pacific Journal of Mathematics}

\section{Vol. 11, No. $1 \quad$ November, 1961}

A. A. Albert, Generalized twisted fields ............................ 1

Richard Arens, Operational calculus of linear relations ................... 9

John Herbert Barrett, Disconjugacy of a self-adjoint differential equation of the fourth order ....................................... 25

Paul Richard Beesack, Hardy's inequality and its extensions ............... 39

Julius Rubin Blum and David Lee Hanson, On invariant probability measures.

II .............................................

Robert Allen Bonic, Symmetry in group algebras of discrete groups.......... 73

R. Creighton Buck, Multiplication operators ...................... 95

Jack Gary Ceder, Some generalizations of metric spaces ................. 105

Meyer Dwass, Random crossings of cumulative distribution functions ......... 127

Albert Edrei, Wolfgang H. J. Fuchs and Simon Hellerstein, Radial distribution and

deficiencies of the values of a meromorphic function ............... 135

William Cassidy Fox, Harmonic functions with arbitrary local singularities ..... 153

Theodore Thomas Frankel, Manifolds with positive curvature ............... 165

Avner Friedman, A strong maximum principle for weakly subparabolic

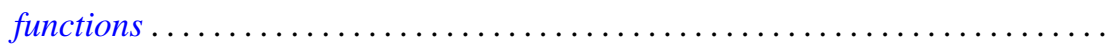

Watson Bryan Fulks and J. O. Sather, Asymptotics. II. Laplace's method for

multiple integrals ......................................

Adriano Mario Garsia and Eugene Richard Rodemich, An embedding of Riemann

surfaces of genus one ..................................... 193

Irving Leonard Glicksberg, Weak compactness and separate continuity......... 205

Branko Grünbaum, On a conjecture of H. Hadwiger .................. 215

Frank J. Hahn, On the action of a locally compact group on $E_{n} \ldots \ldots \ldots \ldots \ldots . . \ldots 221$

Magnus R. Hestenes, Relative hermitian matrices ..................... 225

G. K. Kalisch, On similarity invariants of certain operators in $L_{p} \ldots \ldots \ldots \ldots .247$

Yitzhak Katznelson and Walter Rudin, The Stone-Weierstrass property in Banach

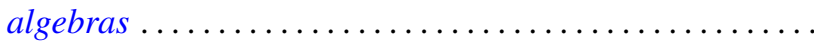

Samir A. Khabbaz, The subgroups of a divisible group $G$ which can be represented as intersections of divisible subgroups of $G \ldots \ldots \ldots \ldots \ldots \ldots \ldots \ldots \ldots . \ldots \ldots 7$

Marvin Isadore Knopp, Construction of a class of modular functions and forms .......................................... 275

Charles Alan McCarthy, Commuting Boolean algebras of projections .......... 295

T. M. MacRobert, Transformations of series of E-functions ................ 309

Heinz Renggli, An inequality for logarithmic capacities ................. 313

M. S. Robertson, Applications of the subordination principle to univalent functions .......................................... 315

David Sachs, Partition and modulated lattices ..................... 325

Frank S. Scalora, Abstract martingale convergence theorems ............... 347

Elbert A. Walker, Torsion endomorphic images of mixed Abelian groups ........ 375

Morgan Ward, The prime divisors of Fibonacci numbers................. 379

Charles R. B. Wright, On the nilpotency class of a group of exponent four....... 387 